\title{
The Progression of Atrophic Gastritis to Gastric Cancer: A Retrospective Study in 61,810 Cases
}

\author{
Yi-Ren $\mathrm{Hu}^{1 *}$, Ying $\mathrm{Yu}^{1 *}$, Jian-Xin $\mathrm{Li}^{1}$, Wei-Chun $\mathrm{Lu}^{1}$, Qi-Jin Shu ${ }^{2}$, Ying-Cong Yu ${ }^{1,2 \#}$ \\ ${ }^{1}$ Wenzhou People's Hospital, the Third Clinical Institute Affiliated to Wenzhou Medical University, Wenzhou, China \\ ${ }^{2}$ The First Affiliated Hospital of Zhejiang Chinese Medical University, Hangzhou, China \\ Email: ${ }^{\sharp} 784038608 @ q q . c o m$
}

How to cite this paper: Hu, Y.-R., Yu, Y., Li, J.-X., Lu, W.-C., Shu, Q.-J. and Yu, Y.-C. (2017) The Progression of Atrophic Gastritis to Gastric Cancer: A Retrospective Study in 61,810 Cases. Journal of Cancer Therapy, 8, 553-560.

https://doi.org/10.4236/jct.2017.86047

Received: May 3, 2017

Accepted: June 17, 2017

Published: June 20, 2017

Copyright ( 2017 by authors and Scientific Research Publishing Inc. This work is licensed under the Creative Commons Attribution International License (CC BY 4.0).

http://creativecommons.org/licenses/by/4.0/

\begin{abstract}
Background and Aim: Chronic atrophic gastritis is a known precancerous lesion for gastric cancer, with an overall 5 -year survival less than $20 \%$. However, there is few new data describing the progression rate from atrophic gastritis (AG) to gastric cancer in China. We retrospectively analyzed the risk of gastric cancer among patients with AG and aimed to determine the accuracy of endoscopy diagnosis of AG in China. Methods: Clinical features and endoscopic profiles of chronic atrophic gastritis patients from Wenzhou People's Hospital between January 2006 and December 2016 were analyzed retrospectively. Results: There were 61,810 cases analyzed retrospectively. 3641 cases of atopic gastritis were diagnosed by endoscopy, in which 1704 cases were confirmed by pathological biopsy; the diagnostic coincidence rate was $46.80 \%$; 2631 cases were diagnosed as atrophic gastritis by pathological biopsy, in which 927 cases were ignored by endoscopy; the miss diagnosis rate was $35.23 \%$. The progression rate from chronic atrophic gastritis and non-atrophic gastritis to gastric cancer respectively was $0.79 \%$ and $0.43 \%$. The rate of intestinal metaplasia in mild AG, moderate AG and severe AG respectively was $75.63 \%, 86.18 \%$ and $90.32 \%, 3.42 \%, 6.27 \%$ and $5.16 \%$ about dysplasia in three different degree atrophic gastritis. Conclusions: Endoscopy diagnosis and pathology diagnosis of AG were statistically different. Patients with atrophic gastritis have higher risk of developing into gastric cancer, but it needs further investigation. Different degrees of atrophic gastritis have the different proportions of intestinal metaplasia and dysplasia.
\end{abstract}

\section{Keywords}

Atrophic Gastritis, Stomach Neoplasms, Disease Progression, Endoscopy, Diagnosis

*Yi-Ren Hu and Ying Yu contributed equally to this paper; they are joint first authors. 


\section{Introduction}

Chronic atrophic gastritis (CAG) is a chronic inflammation characterized by the reduction or disappearance of the inherent gland of the gastric mucosa. The link between CAG and intestinal type gastric carcinoma was illuminated by Corra $\mathrm{P}$ in 1988, who deemed that human gastric carcinogenesis was an multistep and multifactorial process and proposed a classical model about the progress: normal gastric mucosa $\rightarrow$ superficial gastritis (later renamed non-atrophic gastritis, $\mathrm{NAG}) \rightarrow$ atrophic gastritis $\rightarrow$ small intestinal metaplasia $\rightarrow$ colonic intestinal metaplasia $\rightarrow$ mild dysplasia $\rightarrow$ severe dysplasia $\rightarrow$ adenocarcinoma [1] [2]. Although the incidence and mortality of gastric cancer in China are falling, in line with a worldwide decrease in this condition [3], the National Central Cancer Registry [4] reported gastric cancer is still the third leading cause of death in China followed by lung cancer and liver cancer. One may estimate that millions of premature deaths may occur annually worldwide due to cancer and ulcer as sequelae of the chronic gastritis [5]. Surveilling and managing atrophic gastritis patients appear to be an effective mean to decrease the mortality of gastric cancer through early detection and treatment. Great attention has been focused on CAG, an acknowledged premalignant gastric lesion. Because most of the clinical symptoms of AG are insidious or lack of specificity, the data available to quantify the gastric cancer risk in patients with AG are very limited and inconsistent. It is reported that the annual incidence ranged from $0 \%$ to $0.2 \%$ [6]. On the other hand, the fare of malignancy strongly guides clinical practices and decision-making, whereby an immediate endoscopy is recommended in elderly people (more than 45 - 55 years of age) with dyspeptic symptoms by most of international and local consensus statement [7]. This will certainly bring patients additional psychological and economic burden. Hence, further researches are badly needed to supplement the database. In this study, clinical data for patients with AG diagnosed at the Digestive Endoscopy Center of the Wenzhou People's Hospital in the past 11 years were retrospectively analyzed. We investigated the progression rates of chronic atrophic gastritis to gastric cancer in China population, and evaluated coincidence rate of endoscopic diagnosis of atrophic gastritis, with trying to provide a basis for decisions on gastric cancer surveillance practice in China populations.

\section{Methods}

\subsection{Endoscopic Database}

In the research database we compiled 92,438 records relating to 61,810 people who had undergone at least one endoscopic examination with stomach biopsy in Wenzhou People's Hospital between January 2006 and December 2016. The Ethics Committee of Wenzhou Medical University, to which the Wenzhou People's Hospital is affiliated, approved the protocol. Each record concludes completed information of the patients' basic information, endoscopy and pathological detail reports. 


\subsection{Inclusion Criteria}

1) atrophic gastritis (AG), which is defined as loss of glandular tissue with and without metaplasia; 2) superficial gastritis(now called non-atrophic gastritis, NAG); 3) further, the data were respectively extracted from the patients with AG, who were associated with intestinal metaplasia (IM), or combined with dysplasia; 4) at the same time, the data of AG evolved into carcinoma and NAG evolved into carcinoma were respectively extracted.

In accordance with the Updated Sydney System [8] [9], the degrees of dysplasia and non-atrophic gastritis were respectively classified into four grades: $0=$ none, 1 = mild, 2 = moderate, and $3=$ severe. Intestinal metaplasia and dysplasia were also classified into four grades as follows: $0=$ none, $1=$ mild, $2=$ moderate, and $3=$ severe.

There are three main routines to analyze the data meeting inclusion criteria (Figure 1):

To compare the diagnose coincidence of CAG between endoscopy and pathological biopsy, we extracted two groups data from patients completed pathological biopsy examination with endoscopy. One group is CAG defined by endoscopy, but performed NAG under the microscope. The other group is just the opposite.

All of CAG diagnosed by biopsy as a study group, at the same time, all nonatrophic gastritis were regarded the control group. We retrospectively review those patients' whole endoscopic data to find how many individuals developed to cancer before his or her last examination.

According to the Updated Sydney System mentioned above, we classified chronic atrophic gastritis into three grades: mild, moderate and severe CAG to count quantities of those three different degrees atrophy accompanied by intestinal metaplasia or dysplasia.

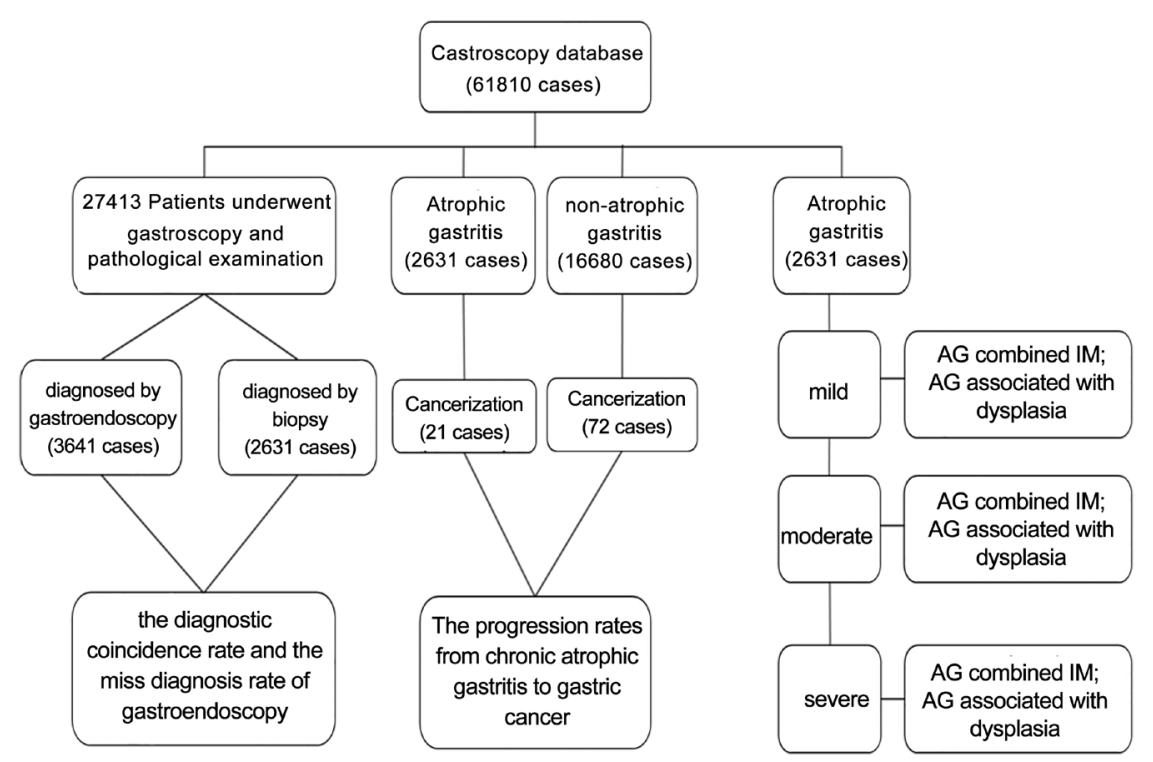

Figure 1. Study population, study aims and results of progress rates, AG, atrophic gastritis; IM, intestinal metaplasia. 


\subsection{Statistical Analysis}

SPSS 17.0 statistical package for Windows was used for the statistical analysis. Comparisons of continuous variables were made by using the student's t test.

\section{Results}

There were 61,810 patients recruited, in which $51.62 \%$ were male with median age of 48.51 years and $48.38 \%$ female with median age of 45.53 years (Table 1 ).

27,413 patients have underwent endoscopy and pathological examination, 3,641 cases of atrophic gastritis were diagnosed by endoscopy, in which 1,704 cases were confirmed by pathological biopsy, the diagnostic coincidence rate was $46.80 \%$; 2,631 cases were diagnosed as atrophic gastritis by pathological biopsy, in which 927 cases were ignored by endoscopy, the miss diagnosis rate was $35.23 \%$. Endoscopy diagnosis and pathology diagnosis of AG were statistically different $(p<0.001)$ (Table 2$)$.

There were 2,631 patients of atrophic gastritis who were diagnosed by pathological biopsy, of whom 21 developed into gastric cancer; Control group, consisted by 16,680 non-atrophic gastritis, of whom 72 patients developed into gastric cancer. Generally speaking, the progression rates from chronic atrophic gastritis and non-atrophic gastritis to gastric cancer respectively was $0.79 \%$ and $0.43 \%$. The result showed no significant statistic difference $(p=0.283)$ (Table 3$)$.

The rate of intestinal metaplasia in mild AG, moderate AG and severe AG respectively was $75.63 \%, 86.18 \%$ and $90.32 \%(p<0.001) ; 3.42 \%, 6.27 \%$ and $5.16 \%$ about dysplasia in three different degree atrophic gastritis $(p=0.004)$. The rate

Table 1. The socio-demographic characteristics of study participants $(n=61,810)$.

\begin{tabular}{ccc}
\hline Gender & Proportion & Median age \\
\hline Man & $51.62 \%$ & 48.51 \\
Women & $48.38 \%$ & 45.53 \\
\hline
\end{tabular}

Table 2. Diagnosis of atrophic gastritis.

\begin{tabular}{ccccc}
\hline Groups & AG & NAG & total & $P$ Value \\
\hline endoscopy & $3,641(5.89 \%)$ & $58,169(94.14 \%)$ & 61,810 & \\
biopsy & $2,631(9.68 \%)$ & $24,543(90.32 \%)$ & 27,174 & $p<0.001$ \\
total & 6272 & 82,712 & 88,984 & \\
\hline
\end{tabular}

AG, atrophic gastritis; NAG, not atrophic gastritis. $P<0.001$.

Table 3. Cancerization of AG and non-atrophic gastritis.

\begin{tabular}{ccccc}
\hline \multicolumn{5}{c}{ Cancerization } \\
& Yes & No & Total & $P$ Value \\
\hline atrophy gastritis & $21(0.79 \%)$ & 2,610 & 2,631 & \\
non-atrophic gastritis & $72(0.43 \%)$ & 11,608 & 11,680 & $p=0.283$ \\
\hline
\end{tabular}


of intestinal metaplasia in mild non-atrophic gastritis, moderate non-atrophic gastritis and severe non-atrophic gastritis respectively was $24.24 \%, 33.93 \%$ and $27.88 \%(p<0.001) ; 1.34 \%, 1.53 \%$ and $1.47 \%$ about dysplasia in three different degree atrophic gastritis $(p=0.687)$ (Table 4 , Table 5$)$.

The rate of intestinal metaplasia in AG group and non-atrophic gastritis group was $80.19 \%$ and $43.07 \%$ respectively $(p<0.001)$. The rate of dysplasia in AG group and non-atrophic gastritis group was $4.52 \%$ and $2.05 \%$ respectively ( $p$ $<0.001$ ) (Table 6).

\section{Conclusion}

Endoscopy diagnosis and pathology diagnosis of AG were statistically different. Patients with atrophic gastritis have higher risk of developing into gastric cancer, but needs further investigation. Different degrees of atrophic gastritis with the proportions of intestinal metaplasia and dysplasia different, the follow-up of atrophic gastritis patients is essential.

Table 4. The rate of IM and dysplasia in different degree of AG.

\begin{tabular}{ccccccccc}
\hline AG & \multicolumn{3}{c}{ combined IM } & \multicolumn{3}{c}{ combined dysplasia } \\
& Yes & No & Total & $P$ Value & Yes & No & Total & $P$ Value \\
\hline total & 2,110 & 521 & 2,631 & $P<0.001$ & 119 & 2,512 & 2,631 & $p=0.004$ \\
& 1,173 & 378 & 1,551 & & 53 & 1,498 & 1,551 & \\
mild AG & $(75.63 \%)$ & $(24.37 \%)$ & & $(3.42 \%)$ & $(96.59 \%)$ & & \\
moderate & 797 & 128 & 925 & 58 & 867 & 925 \\
AG & $(86.16 \%)$ & $(13.84 \%)$ & & $(6.27 \%)$ & $(93.73 \%)$ & & \\
severe AG & 140 & 15 & 155 & 8 & 147 & 155 \\
& $(90.32 \%)$ & $(9.68 \%)$ & & $(5.16 \%)$ & $(94.84 \%)$ & & \\
\hline
\end{tabular}

AG, atrophic gastritis; IM, intestinal metaplasia. All diagnosis referred to histodiagnosis.

Table 5. The rate of IM and dysplasia in different degree of non-atrophic gastritis.

\begin{tabular}{|c|c|c|c|c|c|c|c|c|}
\hline \multirow{2}{*}{$\begin{array}{c}\text { non-atrophic } \\
\text { gastritis }\end{array}$} & \multicolumn{4}{|c|}{ combined IM } & \multicolumn{4}{|c|}{ combined dysplasia } \\
\hline & Yes & No & Total & $P$ Value & Yes & No & Total & $P$ Value \\
\hline total & 5,030 & 11,650 & 11,680 & $p<0.001$ & 239 & 16,441 & 11,680 & $p=0.687$ \\
\hline mild & $\begin{array}{c}1,915 \\
(24.24 \%)\end{array}$ & $\begin{array}{c}5,382 \\
(73.76 \%)\end{array}$ & 7,297 & & $\begin{array}{c}98 \\
(1.34 \%)\end{array}$ & $\begin{array}{c}7199 \\
(98.66 \%)\end{array}$ & 7,297 & \\
\hline moderate & $\begin{array}{c}2,793 \\
(33.95 \%)\end{array}$ & $\begin{array}{c}5,435 \\
(66.05 \%)\end{array}$ & 8,228 & & $\begin{array}{c}124 \\
(1.51 \%)\end{array}$ & $\begin{array}{c}8,104 \\
(98.49 \%)\end{array}$ & 8,228 & \\
\hline severe & $\begin{array}{c}322 \\
(27.88 \%)\end{array}$ & $\begin{array}{c}833 \\
(72.12 \%)\end{array}$ & 1,155 & & $\begin{array}{c}17 \\
(1.47 \%)\end{array}$ & $\begin{array}{c}1,138 \\
(98.53 \%)\end{array}$ & 1,155 & \\
\hline
\end{tabular}

Table 6. The rate of IM and dysplasia in non-atrophic and atrophy gastritis.

\begin{tabular}{cccc}
\hline & atrophy gastritis & non-atrophic gastritis & $P$ Value \\
\hline combined IM & $2,110(80.19 \%)$ & $5,030(43.07 \%)$ & $p<0.001$ \\
combined dysplasia & $119(4.52 \%)$ & $239(2.05 \%)$ & $p<0.001$ \\
\hline
\end{tabular}




\section{Discussion}

A large-scale population-based cohort study, with a 13-year follow-up in Japan found that gastric screening can significantly reduce the mortality of gastric cancer [10]. So the vigilant follow-up in the high risk groups, such as atrophic gastritis populations, is an effective strategy for improving the bad condition of gastric cancer. In Western countries, AG is generally observed in histological examination of random biopsies obtained during endoscopy; whereas in Asian countries including China, the presence and extension of AG are frequently observed by endoscopy [11]. From our conclusions, the accuracy of endoscopy diagnosis of atrophic gastritis is low, only $46.80 \%$, and the miss diagnosis rate is high, up to $35.23 \%$. How to improve the endoscopy diagnosis of atrophic gastritis? Research [12] has shown that gastric xanthelasma(s) is a warning endoscopic sign for the presence of AG and advanced intestinal metaplasia. However, in our study, we haven't found any gastric xanthelasma(s). There exists another non-invasive measure to assess CAG, which is serum PGI and PGII analysis [13] [14]. According to a study in Sweden [15], serological and histological testing for atrophic gastritis showed $96 \%$ agreement. Another important result we have found was that the progression rate of atrophic gastritis to gastric cancer $(0.79 \%)$ was higher than that of non-atrophic gastritis $(0.43 \%)$, consistent with the Correa $P$ pattern. But the difference was not significant; this may due to the sample and the multiple choices of patients to undergo the procedure. There are several hospitals available for AG patient follow-up, and the loss ratio of follow-up is more likely higher in AG patients than which in non-atrophic gastritis participants. Chooi EY et al. [16] conducted a retrospective analysis involving 1592 patients who underwent endoscopy during 1985-2009 at Renji Hospital, Shanghai, China. One of their results is that $1.44 \%$ patients presented with gastric cancers resulting from CAG. The lower incidence in our study may be caused by the shorter follow-up periods. On the other hand, with the degree increased in atrophy, intestinal metaplasia $(75.63 \%, 86.16 \%, 90.32 \%)(p<0.001)$ and dysplasia $(3.42 \%, 6.27 \%, 5.16 \%)(p=0.004)$ also showed an increasing trend, suggesting that a raise in the degree of atrophy may lead to an increased risk of gastric cancer. Correspondingly, patients with a high level non-atrophic gastritis show a high risk of combining $\operatorname{IM}(p<0.001)$ but the significance of different degree non-atrophic gastritis combined dysplasia was low $(p=0.687)$. Moreover, compared with non-atrophic gastritis, AG has higher rate of IM $(p<0.001)$ and dysplasia $(p<0.001)$. Many researches have illuminated the progressive process of CAG to gastric cancer, an observational cohort study [17] established in Sweden also showed that all stages of Correa's cascade predicted an incidence of gastric cancer. Their observed progression rate approximately $1.17 \%$ with gastritis, $2 \%$ with atrophic gastritis within 20 years among patients who undergo gastroscopy with biopsy for clinical indications. Qiang $Z$ et al. [18] found that the incidence of dysplasia of atrophic gastritis patients with a course more than 10 years as high as $42.4 \%$. Therefore, the monitoring and early intervention of precancerous lesions, especially atrophic gastritis, are important for reducing the incidence of 
gastric cancer. Our study further validated the occurrence of gastric cancer which is a multistep process and follow-up to AG patients is essential. As our research is a retrospective study, there is some unavoidable bias in the findings, such as, patients may choose several hospitals for treatment during the eleven years, and we just analyzed data in our hospital. Further study of large sample and in multiple centers should be organized.

\section{References}

[1] Correa, P. (1988) A Human Model of Gastric Carcinogenesis. Cancer Research, 48, 3554-3560.

[2] Correa, P. (1992) Human Gastric Carcinogenesis: A Multistep and Multifactorial Process-First American Cancer Society Award Lecture on Cancer Epidemiology and Prevention. Cancer Research, 52, 6735-6740.

[3] Chen, W., Zheng, R., Zhang, S., et al. (2014) Annual Report on Status of Cancer in China, 2010. Chinese Journal of Cancer Research, 26, 48-58.

[4] McGuire, S. (2016) World Cancer Report 2014. Geneva, Switzerland: World Health Organization, International Agency for Research on Cancer, WHO Press, 2015. Advances in Nutrition, 7, 418-419. https://doi.org/10.3945/an.116.012211

[5] Sipponen, P. and Maaroos, H.I. (2015) Chronic Gastritis. Scandinavian Journal of Gastroenterology, 50, 657-667.

[6] de Vries, A.C., van Grieken, N.C., Looman, C.W., et al. (2008) Gastric Cancer Risk in Patients with Premalignant Gastric Lesions: A Nationwide Cohort Study in the Netherlands. Gastroenterology, 134, 945-952.

https://doi.org/10.1053/j.gastro.2008.01.071

[7] Sipponen, P. and Graham, D.Y. (2007) Importance of Atrophic Gastritis in Diagnostics and Prevention of Gastric Cancer: Application of Plasma Biomarkers. Scandinavian Journal of Gastroenterology, 42, 2-10. https://doi.org/10.1080/00365520600863720

[8] Dixon, M.F., Genta, R.M., Yardley, J.H. and Correa, P. (1996) Classification and Grading of Gastritis. The Updated Sydney System. International Workshop on the Histopathology of Gastritis, Houston 1994. The American Journal of Surgical Pathology, 20, 1161-1181. https://doi.org/10.1097/00000478-199610000-00001

[9] Stolte, M. and Meining, A. (2001) The Updated Sydney system: Classification and Grading of Gastritis as the Basis of Diagnosis and Treatment. Canadian Journal of Gastroenterology, 15, 591-598. https://doi.org/10.1155/2001/367832

[10] Lee, K.J., Inoue, M., Otani, T., et al. (2006) Gastric Cancer Screening and Subsequent Risk of Gastric Cancer: A Large-Scale Population-Based Cohort Study, with a 13-Year Follow-Up in Japan. International Journal of Cancer, 118, 2315-2321. https://doi.org/10.1002/ijc.21664

[11] Joo, Y.E., Park, H.K., Myung, D.S., et al. (2013) Prevalence and Risk Factors of Atrophic Gastritis and Intestinal Metaplasia: A Nationwide Multicenter Prospective Study in Korea. Gut and liver, 7, 303-310. https://doi.org/10.5009/gnl.2013.7.3.303

[12] Koksal, A.S., Suna, N., Kalkan, I.H., et al. (2016) Is Gastric Xanthelasma an Alarming Endoscopic Marker for Advanced Atrophic Gastritis and Intestinal Metaplasia? Digestive Diseases and Sciences, 61, 2949-2955. https://doi.org/10.1007/s10620-016-4210-6

[13] Juan, C.W., Yin, L., Kang, Q., Chen, Z.Z., Liang, W.S. and Cheng, J. (2017) The Serum Pepsinogen Test as a Predictor of Kazakh Gastric Cancer. Scientific Reports, 7, 
Article No. 43536. https://doi.org/10.1038/srep43536

[14] Varis, K., Sipponen, P., Laxen, F., et al. (2000) Implications of Serum Pepsinogen I in Early Endoscopic Diagnosis of Gastric Cancer and Dysplasia. Helsinki Gastritis Study Group. Scandinavian Journal of Gastroenterology, 35, 950-956. https://doi.org/10.1080/003655200750023011

[15] Storskrubb, T., Aro, P., Ronkainen, J., et al. (2008) Serum Biomarkers Provide an Accurate Method for Diagnosis of Atrophic Gastritis in a General Population: The Kalixanda Study. Scandinavian Journal of Gastroenterology, 43, 1448-1455. https://doi.org/10.1080/00365520802273025

[16] Chooi, E.Y., Chen, H.M., Miao, Q., et al. (2012) Chronic Atrophic Gastritis Is a Progressive Disease: Analysis of Medical Reports from Shanghai (1985-2009). Singapore Medical Journal, 53, 318-324.

[17] Song, H., Ekheden, I.G., Zheng, Z., Ericsson, J., Nyren, O. and Ye, W. (2015) Incidence of Gastric Cancer among Patients with Gastric Precancerous Lesions: Observational Cohort Study in a Low Risk Western Population. BMJ, 351, h3867. https://doi.org/10.1136/bmj.h3867

[18] Zhang, Q., Lian, Z., Wang, L., et al. (2012) Analysis of Alarming Signals for the Progression of Atrophic Gastritis to Dysplasia. Revista Espanola De Enfermedades Digestivas, 104, 399-404. https://doi.org/10.4321/S1130-01082012000800002

Submit or recommend next manuscript to SCIRP and we will provide best service for you:

Accepting pre-submission inquiries through Email, Facebook, LinkedIn, Twitter, etc. A wide selection of journals (inclusive of 9 subjects, more than 200 journals)

Providing 24-hour high-quality service

User-friendly online submission system

Fair and swift peer-review system

Efficient typesetting and proofreading procedure

Display of the result of downloads and visits, as well as the number of cited articles

Maximum dissemination of your research work

Submit your manuscript at: http://papersubmission.scirp.org/

Or contact jct@scirp.org 